\title{
Transversity in Exclusive and Inclusive Processes
}

\author{
Leonard Gamberg*, Gary R. Goldstein ${ }^{\dagger}$ and Karo A. Oganessyan*** \\ ${ }^{*}$ Division of Science, Penn State-Berks Lehigh Valley College, Reading, PA 19610, USA ${ }^{1}$ \\ ${ }^{\dagger}$ Department of Physics and Astronomy, Tufts University, Medford, MA 02155, USA ${ }^{2}$ \\ ** INFN-Laboratori Nazionali di Frascati, Enrico Fermi 40, I-00044 Frascati, Italy \\ ${ }^{\ddagger}$ DESY, Notkestrasse 85, 22603 Hamburg, Germany
}

\begin{abstract}
Both meson photoproduction and semi-inclusive deep inelastic scattering can potentially probe transversity properties of the nucleon. We explore how that potential can be realized dynamically. The role of rescattering in both exclusive and inclusive meson production as a source for single spin asymmetries is examined. Using a dynamical model, we evaluate the spin independent $\cos 2 \phi$ asymmetry associated with transversity of quarks inside unpolarized hadrons, at HERMES kinematics. We also explore the effects of rescattering on the transversity distribution of the nucleon.
\end{abstract}

\section{Transversity}

It is well known that the leading twist transversity distribution $h_{1}(x)$ [1] and its first moment, the tensor charge, being chiral odd cannot be accessed in deep inelastic scattering. However, $h_{1}(x)$ can be probed when at least two hadrons are present, e.g. Drell Yan [2] or semi-inclusive deep inelastic scattering (SIDIS). In the latter process at leading twist, the property of quark transversity can be measured via the azimuthal asymmetry in the fragmenting hadron's momentum and spin distributions. For example, spinless hadrons produced in the so-called Collins asymmetry [3] depend on the transverse momentum of quarks in the target, $k_{T}$, and fragmentation functions, $p_{T}$ [4]. Including transverse momentum leads to an increase in the number of leading twist distribution and fragmentation functions $\left(e . g . h_{1 T}\left(x, k_{T}\right), h_{1 T}^{\perp}\left(x, k_{T}\right), H_{1}^{\perp}\left(z, p_{T}\right)\right)$. Allowing time reversal odd ( $T$-odd) quark distribution functions [5, 6, 7], $h_{1}^{\perp}\left(x, k_{T}\right), f_{1 T}^{\perp}\left(x, k_{T}\right)$ suggests they enter the semi-inclusive unpolarized momentum, and polarized spin, asymmetries. That is, the distribution $f_{1 T}^{\perp}\left(x, k_{T}\right)$ representing the number density of unpolarized quarks in transversely polarized nucleons, maybe entering the recent measurements of SSAs at HERMES and SMC in semi-inclusive pion electroproduction [8]. Alternatively, $h_{1}^{\perp}\left(x, k_{T}\right)$ which describes the transfer of transversity to quarks inside unpolarized hadrons may enter transverse momentum dependent asymmetries. Beyond the $T$-odd properties, the existence of these distributions are a signal of the essential role played by the intrinsic transverse quark momentum and the corresponding angular momentum of quarks inside the target and fragmenting hadrons in these hard scattering processes.

\footnotetext{
${ }^{1}$ Supported by a Research Development Grant, Penn State Berks. Based on talk given at $15^{\text {th }}$ International Spin Physics Symposium (SPIN 2002), 9-14 September, 2002.

${ }^{2}$ Supported by the US Department of Energy DE-FG02-29ER40702.
} 
Further insight into transversity has come from analyzing quark-target helicity flip amplitudes in deeply virtual Compton scattering (DVCS)[9]. Angular momentum conservation requires that helicity changes are accompanied by transferring 1 or 2 units of orbital angular momentum; again highlighting the essential role of intrinsic $k_{T}$ and orbital angular momentum in determining transversity.

\section{The Exchange Picture}

The interdependence of transversity on quark orbital angular momentum and $k_{T}$ is more general than suggested in the above discussion on SSAs and the GPD analysis of transversity. This behavior arises in ref. [10] where we study the vertex function associated with the tensor charge in exclusive meson production. Again, angular momentum conservation results in the transfer of orbital angular momentum $\ell=1$ carried by the dominant $J^{P C}=1^{+-}$mesons to compensate for the non-conservation of helicity across the vertex. Transverse momentum dependence arises from the axial vector mesons that dominate the tensor coupling (They are the $C$-odd $-h_{1}(1170), h_{1}(1380), b_{1}(1235)$ ). These mesons are in the $(35 \otimes \ell=1)$ multiplet of the $S U(6) \otimes O(3)$ symmetry group that best represents the mass symmetry among the low lying mesons. Along with axial vector dominance this symmetry results in the isoscalar and isovector contribution to the tensor charge

$$
\delta u\left(\mu^{2}\right)-\delta d\left(\mu^{2}\right)=\frac{5}{6} \frac{g_{A}}{g_{V}} \frac{M_{a_{1}}^{2}}{M_{b_{1}}^{2}} \frac{\left\langle k_{T}^{2}\right\rangle}{M_{N} M_{b_{1}}}, \quad \delta u\left(\mu^{2}\right)+\delta d\left(\mu^{2}\right)=\frac{3}{5} \frac{M_{b_{1}}^{2}}{M_{h_{1}}^{2}} \delta q^{v} .
$$

Each depends on two powers of the average intrinsic quark momentum $\left\langle k_{T}^{2}\right\rangle$, because the tensor couplings involve helicty flips associated with kinematic factors of 3-momentum transfer. as required by angular momentum conservation.

The $k_{T}$ dependence can be understood on fairly general grounds from the kinematics of the exchange picture in exclusive pseudoscalar meson photoproduction. For large $s$ and relatively small momentum transfer $t$ simple combinations of the four helicity amplitudes involve definite parity exchanges. The four independent helicity amplitudes can have the minimum kinematically allowed powers,

$$
f_{1}=f_{1+, 0+} \propto k_{T}^{1}, \quad f_{2}=f_{1+, 0-} \propto k_{T}^{0}, \quad f_{3}=f_{1-, 0+} \propto k_{T}^{2}, \quad f_{4}=f_{1-, 0-} \propto k_{T}^{1} .
$$

However, in single hadron exchange (or Regge pole exchange) parity conservation requires $f_{1}= \pm f_{4}$ and $f_{2}=\mp f_{3}$ for even/odd parity exchanges. These pair relations, along with a single hadron exchange model, force $f_{2}$ to behave like $f_{3}$ for small $t$. This introduces the $k_{T}^{2}$ factor into $f_{2}$. However for a non-zero polarized target asymmetry to arise there must be interference between single helicity flip and non-flip and/or double flip amplitudes. Thus this asymmetry must arise from rescattering corrections (or Regge cuts or eikonalization or loop corrections) to single hadron exchanges. That is, one of the amplitudes in

$$
P_{y}=\frac{2 \operatorname{Im}\left(f_{1}^{*} f_{3}-f_{4}^{*} f_{2}\right)}{\sum_{j=1 \ldots 4}\left|f_{j}\right|^{2}}
$$


must acquire a different phase. In fact rescattering reinstates $f_{2} \propto k_{T}^{0}$ by integrating over loop $k_{T}$, which effectively introduces a $\left\langle k_{T}^{2}\right\rangle$ factor [11]. This is true for the inclusive process as well, where only one final hadron is measured; a relative phase in a helicity flip three body amplitude is required.

\section{Rescattering and SIDIS}

Recently a rescattering approach was applied to the calculation of SSA in pion electroproduction, using a QCD motivated quark-diquark model of the nucleon [12] (BHS). In Ref. [13,6] the rescattering effect is interpreted as giving rise to the $T$-odd Sivers [14] $f_{1 T}^{\perp}$ function; the number density of unpolarized quarks in a transversely polarized target. Being $T$-odd, this asymmetry vanishes at tree level. The important lesson beyond the model calculation, is that, theoretically, final state interactions are essential for producing non-zero SSAs. Furthermore, the phenomenological determination of quark spin distributions can be disentangled from measurements of SSAs.

We have investigated the rescattering contributions to the transversity distribution $h_{1}(x)$ and the $T$-odd function $h_{1}^{\perp}(x)$ and corresponding asymmetries in SIDIS. Collins [3] considered one such process, the production of pions from transversely polarized quarks in a transversely polarized target. The corresponding SSA involves the convolution of the transversity distribution function and the $T$-odd fragmentation function, $h_{1}(x) \star H_{1}^{\perp}(z)[15,16]$. The transversity distribution function is defined through the light-cone quark distribution with gauge link indicated,

$$
\begin{aligned}
s_{T}^{i} \Delta f_{T}\left(x, k_{T}\right)= & \frac{1}{2} \sum_{n} \int \frac{d \xi^{-} d^{2} \xi_{\perp}}{(2 \pi)^{3}} e^{-i\left(\xi^{-} k^{+}-\vec{\xi}_{\perp} \vec{k}_{\perp}\right)}\left\langle P\left|\bar{\psi}\left(\xi^{-}, \xi_{\perp}\right)\right| n\right\rangle \\
& \left\langle n\left|\left(-i e_{1} \int_{0}^{\infty} A^{+}\left(\xi^{-}, 0\right) d \xi^{-}\right) \gamma^{+} \gamma^{i} \gamma^{5} \psi(0)\right| P\right\rangle+\text { h.c. },
\end{aligned}
$$

where $e_{1}$ is the charge of the struck quark and $n$ represents intermediate diquark states. Integration over $q^{\mu}$, the gluon momentum we obtain

$$
\begin{aligned}
& s_{T}^{i} \Delta f_{T}\left(x, k_{T}\right)=\frac{e_{1} e_{2} g^{2}}{2(2 \pi)^{4}} \frac{1-x}{\Lambda\left(k_{T}^{2}\right)}\left\{\left(S_{T}^{i}\left[(m+x M)^{2}+k_{T}^{2}\right]+2 k_{T}^{i} \mathbf{S}_{T} \cdot \mathbf{k}_{T}\right)\right. \\
& \left.\times \frac{1}{k_{T}^{2}+\Lambda(0)^{2}+\lambda_{g}^{2}}\left(\ln \frac{\Lambda\left(k_{T}^{2}\right)}{\Lambda(0)}+\ln \frac{k_{T}^{2}+\lambda_{g}^{2}}{\lambda_{g}^{2}}\right)-\left(S_{T}^{i} k_{T}^{2}+2 k_{T}^{i} \mathbf{S}_{T} \cdot \mathbf{k}_{T}\right) \frac{1}{k_{T}^{2}} \ln \frac{\Lambda\left(k_{T}^{2}\right)}{\Lambda(0)}\right\},
\end{aligned}
$$

where $\Lambda\left(k_{T}^{2}\right)=k_{T}^{2}+x(1-x)\left(-M^{2}+\frac{m^{2}}{x}+\frac{\lambda^{2}}{1-x}\right)$. The (Abelian) gluon mass (usually chosen at $\left.\lambda_{g} \approx 1 \mathrm{GeV}\right)$ is indicative of $\chi S B$ scale and appears here to regulate the IR divergence. This one loop contribution constitutes the next order term in an eikonalization. The first part has the same nucleon spin dependent structure as a tree level model calculation (modified by the log terms) - it is leading twist and a combination of $h_{1 T}\left(x, k_{T}\right)$ and $h_{1 T}^{\perp}\left(x, k_{T}\right)$. The second part has a different structure than tree level - it appears as a rescattering effect only. It is IR finite and, in this model, is proportional to the one loop result for $f_{1 T}^{\perp}$ [13] and $\mathscr{P}_{y}$ in BHS. The ratio of $h_{1 T}\left(x, k_{T}\right)$ to $h_{1 T}^{\perp}\left(x, k_{T}\right)$ will differ 

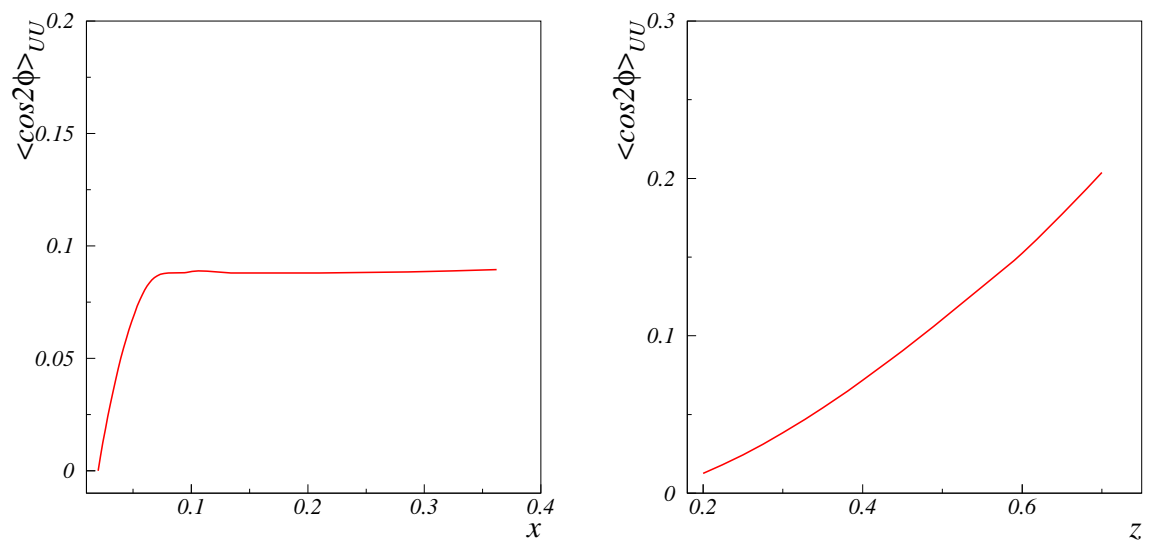

FIGURE 1. Left Panel: The $\langle\cos 2 \phi\rangle_{U U}$ asymmetry for $\pi^{+}$production as a function of $x$. Right Panel: The $\langle\cos 2 \phi\rangle_{U U}$ asymmetry for $\pi^{+}$production as a function of $z$.

from the tree level. When combined with a measure of transversely polarized quarks, the fragmentation function $H_{1}^{\perp}(z)$, the integrated $h_{1}(x)$ will contribute to the observable weighted meson azimuthal asymmetry from a transversely polarized nucleon $[4,16]$.

As mentioned in the introduction, the $T$-odd structure function $h_{1}^{\perp}\left(x, k_{T}\right)$ is of great interest theoretically, since it vanishes at tree level, and experimentally, since its determination does not involve polarized nucleons. Repeating the calculation above without nucleon polarization leads to the result [17]

$$
\frac{\varepsilon_{+-\perp j} k_{T j}}{M} h_{1}^{\perp}\left(x, k_{T}\right)=\frac{e_{1} e_{2} g^{2}}{2(2 \pi)^{4}} \frac{(m+x M)(1-x)}{\Lambda\left(k_{T}^{2}\right)} \varepsilon_{+-\perp j} k_{T j} \frac{1}{k_{T}^{2}} \ln \frac{\Lambda\left(k_{T}^{2}\right)}{\Lambda(0)} .
$$

It is leading twist and IR finite. Being $T$-odd it will appear in SIDIS observables along with $T$-odd fragmentation functions. In particular, the following weighted semiinclusive DIS cross section projects out a leading $\cos 2 \phi$ asymmetry [16],

$$
\left\langle\frac{\left|P_{h \perp}^{2}\right|}{M M_{h}} \cos 2 \phi\right\rangle_{U U}=\frac{\int d^{2} P_{h \perp} \frac{\left|P_{h \perp}^{2}\right|}{M M_{h}} \cos 2 \phi d \sigma}{\int d^{2} P_{h \perp} d \sigma}=\frac{8(1-y) \sum_{q} e_{q}^{2} h_{1}^{\perp(1)}(x) z^{2} H^{\perp(1)}(z)}{\left(1+(1-y)^{2}\right) \sum_{q} e_{q}^{2} f_{1}(x) D_{1}(z)}
$$

where the subscript $U U$ indicates unpolarized beam and target(Note: The non-vanishing $\cos 2 \phi$ asymmetry originating from kinematical and dynamical effects only appears at order $\left.1 / Q^{2}[18,4,19]\right)$. The functions $h_{1}^{\perp(1)}(x)$ and $H_{1}^{\perp(1)}(z)$ are the weighted moments of the distribution and fragmentation functions

$$
h_{1}^{\perp(1)}(x) \equiv \int d^{2} k_{T} \frac{k_{T}^{2}}{2 M^{2}} h_{1}^{T}\left(x, k_{T}\right), \quad H_{1}^{\perp(1)}(z) \equiv z^{2} \int d^{2} p_{T} \frac{k_{T}^{2}}{2 M_{h}^{2}} H_{1}^{T}\left(z,-p_{T}\right) .
$$

$k_{T}, p_{T}$, are the transverse momentum of the quark in the target proton, and fragmenting quark respectively and $M, M_{h}$ are the mass of the target proton and produced hadron. We evaluate the $\langle\cos 2 \phi\rangle_{U U}$ asymmetry obtained from the approximation,

$$
\langle\cos 2 \phi\rangle_{U U} \approx \frac{M M_{h}}{\left\langle P_{h \perp}^{2}\right\rangle}\left\langle\frac{\left|P_{h \perp}^{2}\right|}{M M_{h}} \cos 2 \phi\right\rangle_{U U}
$$


in the HERMES kinematic range corresponding to $1 \mathrm{GeV}^{2} \leq Q^{2} \leq 15 \mathrm{GeV}^{2}, 4.5$ $\mathrm{GeV} \leq E_{\pi} \leq 13.5 \mathrm{GeV}, 0.2 \leq z \leq 0.7,0.2 \leq y \leq 0.8$, and taking $\left\langle P_{h \perp}^{2}\right\rangle=0.25 \mathrm{GeV}^{2}$ as input. The Collins ansatz [3,4] for the analyzing power of transversely polarized quark fragmentation function $H_{1}^{\perp(1)}(z)$, has been adopted [20]. For $D_{1}(z)$, the simple parameterization from Ref. [21] was used. In Fig1 the $\langle\cos 2 \phi\rangle_{U U}$ of Eq.(6) for $\pi^{+}$ production on a proton target is presented as a function of $x$ and $z$, respectively. Using $\Lambda_{Q C D}=0.2 \mathrm{GeV}$ and $\mu=0.8 \mathrm{GeV}$, Fig. 1 indicates that the $\cos 2 \phi$ asymmetry related to is large enough (about $8 \%$ ) to be measured [22].

\section{Conclusions}

The interdependence of intrinsic transverse quark momentum and angular momentum conservation are intimately tied with the studies of transversity. This is demonstrated from analyses of the tensor charge in the context of the vector dominance approach to exclusive meson photo-production, to SSAs in SIDIS[10]. In the study of the tensor charge we find $\left\langle k_{T}^{2}\right\rangle$ factor that appears in rescattering models in meson photoproduction where interference phenomena are non-zero due to rescattering. In the case of unpolarized beam and target we have predicted at HERMES energies the sizable $\cos 2 \phi$ asymmetry associated with the asymmetric distributions of transversely polarized quarks inside of unpolarized hadrons.

\section{Acknowledgements}

L.G. thanks the organizers of SPIN 2002 for the invitation to present this work. I also thank Daniel Boer, Dennis Sivers and Feng Yuan for useful discussions.

\section{REFERENCES}

1. X. Artu and M. Mekhfi, Z. Phys. C45 (1990) 669; R. L. Jaffe and X. Ji, Phys. Rev. Lett. 67 (1991) 552; Nucl. Phys. B375 (1992) 527.

2. J. Ralston and D. E. Soper, Nucl. Phys. B152 (1979) 109.

3. J.C. Collins, Nucl. Phys. B396, 161 (1993).

4. A. M. Kotzinian, Nucl. Phys B441 (1995) 234.

5. M. Anselmino and F. Murgia, Phys. Lett B442 (1998) 470; M. Anselmino, V. Barone, A. Drago and F. Murgia, hep-ph/0209073.

6. J. C. Collins, Phys. Lett. B536 (2002) 43.

7. R. D. Tangerman and P. J. Mulders, Phys. Lett. B352 (1995) 129; Phys. Rev. D51 (1995) 3357; Nucl. Phys. B461 (1996) 197.

8. A. Airapetian et al., Phys. Rev. Lett. 84 (2000) 4047; A. Bravar, Nucl. Phys. Proc. Suppl. 79 (1999) 520.

9. P. Hoodbhoy and X. Ji, Phys. Rev. D58 (1998) 054006; M. Diehl, Eur. Phys. J. C19 (2001) 485.

10. L. Gamberg and G. R. Goldstein, Phys. Rev. Lett. 87 (2001) 242001.

11. G. R. Goldstein and J. F. Owens, Phys. Rev. D7 (1973) 865; Nucl. Phys. B71 (1974) 461.

12. S. Brodsky, D.S. Hwang and I. Schmidt, Phys. Lett. B530 (2002) 99.

13. X. Ji and F. Yuan, Phys. Lett. B543 (2002) 66; A.V. Belitsky, et al., hep-ph/0208038.

14. D. Sivers, Phys. Rev D 41 (1990) 83 ; Phys. Rev. D 43 (1991) 261.

15. A. M. Kotzinian and P. J. Mulders, Phys. Lett. B406 (1997) 373.

16. D. Boer and P. J. Mulders, Phys. Rev. D57 (1998) 5780. 
17. G. R. Goldstein and L. Gamberg, hep-ph/0209085.

18. R.N. Cahn, Phys. Lett. B78 (1978) 269; Phys. Rev. D40 (1989) 3107.

19. K.A. Oganessyan, et.al., Eur. Phys. J. C5 (1998) 681.

20. K.A. Oganessyan, N. Bianchi, E. De Sanctis, and W.D. Nowak, Nucl. Phys. A689 (2001) 784.

21. E. Reya, Phys. Rep. 69 (1981) 195.

22. L. Gamberg, G. R. Goldstein and K.A. Oganessyan, in preparation. 